\title{
A Conceptual Model for Assessing the Minimum Size Area for an Area-Wide Integrated Pest Management Program
}

\author{
Hugh J. Barclay, ${ }^{1}$ Robert Matlock, ${ }^{2}$ Stuart Gilchrist, ${ }^{3,4}$ David M. Suckling, ${ }^{5}$ Jesus Reyes, ${ }^{6,7}$ \\ Walther R. Enkerlin, 7,8 and Marc J. B. Vreysen ${ }^{7,9}$
}

${ }^{1}$ Pacific Forestry Centre, 506 West Burnside Road, Victoria, BC, Canada V8Z 1M5

${ }^{2}$ Biology Department, CSI, CUNY, 2800 Victory Boulevard, Staten Island, 10314, USA

${ }^{3}$ Australian Fruit Fly Research Centre, School of Biological Sciences A12, The University of Sydney, NSW 2006, Australia

${ }^{4}$ Evolution and Ecology Research Centre, School of Biological, Earth, and Environmental Sciences,

The University of New South Wales Sydney, NSW 2052, Australia

${ }^{5}$ The New Zealand Institute for Plant and Food Research Ltd., PB 4704, Christchurch 8140, New Zealand

${ }^{6}$ United States Department of Agriculture, 4a. avenida 12-62, Zona 10 Ciudad de Guatemala 01010, Guatemala

${ }^{7}$ Insect Pest Control Section, Joint FAO/IAEA Programme of Nuclear Techniques in Food and Agriculture, International Atomic Energy Agency, P.O. Box 100, Wagramerstrasse 5, 1400 Vienna, Austria

${ }^{8}$ Moscamed Regional Program, 16 Street 3-38 Area 10, Guatemala City, Guatemala

${ }^{9}$ Insect Pest Control Laboratory, Joint FAO/IAEA Division of Nuclear Techniques in Food and Agriculture, International Atomic Energy Agency, P.O. Box 100, Wagramerstrasse 5, 1400 Vienna, Austria

Correspondence should be addressed to Hugh J. Barclay, hbarclay@nrcan.gc.ca

Received 9 December 2010; Accepted 13 January 2011

Academic Editor: Allen V. Barker

Copyright (C) 2011 Hugh J. Barclay et al. This is an open access article distributed under the Creative Commons Attribution License, which permits unrestricted use, distribution, and reproduction in any medium, provided the original work is properly cited.

\begin{abstract}
A conceptual model was developed based on the two basic spatial elements of area-wide integrated pest management (AW-IPM), a core area and a buffer zone, to determine the minimum size of the protected area for the program to be technically feasible and economically justifiable. The model consisted of a biological part (insect dispersal) and an economic part. The biological part used random walks and diffusion equations to describe insect dispersal and to determine the minimum width of the buffer zone required to protect the core area from immigration of pests from outside. In the economic part, the size of the core area was calculated to determine the point at which the revenues from the core area equal the control costs. This model will need to be calibrated and validated for each species and geographic location. Tsetse flies and the Mediterranean fruit fly are used as case studies to illustrate the model.
\end{abstract}

\section{Introduction}

Classical integrated pest management (IPM), which aims at managing pests by the integration of biological, cultural, physical, and chemical tools in a way that minimizes economic, health, and environmental risks [1], has remained a dominant paradigm of pest control for the last 50 years. The different control tactics can be integrated on a field-byfield basis or by using an area-wide approach [2]. Area-wide integrated pest management (AW-IPM) is the integrated use of various control tactics against an entire pest population within a delimited geographical area to achieve economic control [3]. The importance of this approach of "total pest population management" has significantly increased for many pests in the past decades, and it is now generally accepted that AW-IPM leads, in many cases, to more sustainable pest control especially for mobile insects [2]. A powerful AW control tactic is the sterile insect technique (SIT), which over the past decades has become accepted as an efficient and cost-effective part of AW-IPM programs against a selected number of insect pests of veterinary, human health, and agricultural importance [4].

A recurrent concern for pest managers is the minimum size of the target area that needs to be considered for an 


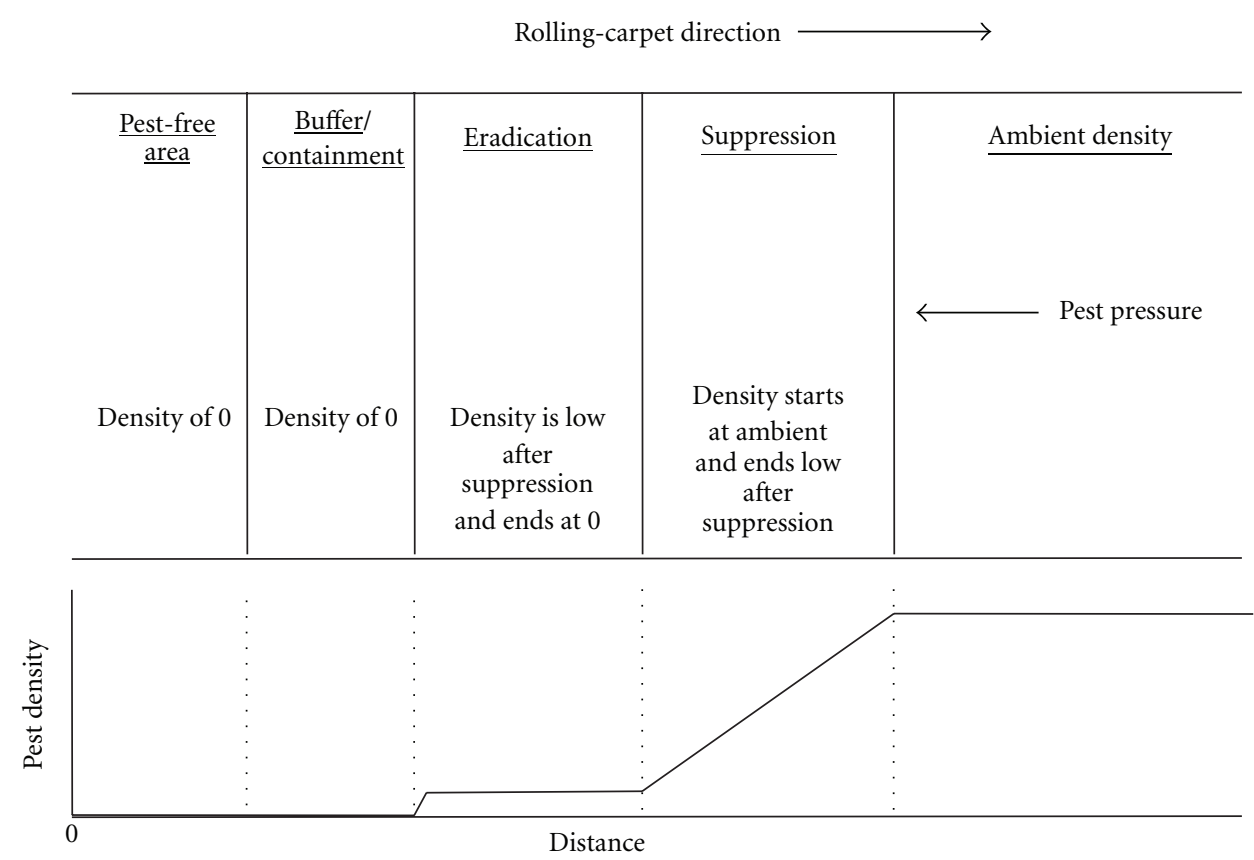

Figure 1: Schematic diagram of the expected changes in pest density from an infested area (high pest pressure), through the buffer zone (B), into the core area $(A)$ in the case of an eradication strategy. In the rolling-carpet approach, declines in pest density represent declines over time, although the form of the slope is schematic.

AW-IPM program to be technically viable and economically justifiable. Due to the lack of adequate practical experience and the absence of models, decisions were sometimes based on educated guesses rather than on sound, scientific principles. Therefore, a conceptual mathematical model was developed that can assist with estimating the minimum area that needs to be considered to successfully apply a series of control tactics according to the AW-IPM approach against insect pests for which there are adequate biological input data. To make the model applicable to a series of pest species amenable to AW-IPM, it was developed in a generic way with a minimum of identified assumptions included. The prototype model creates a basis for a decision-support tool to assess the minimum dimensions of an intervention area required for the establishment of a pest-free area (as described in the International Standard of Requirements for the Establishment of Pest Free Areas (ISPM 4)) or areas of low pest prevalence (as described in the International Standard of Requirements for the Establishment of Areas of Low Pest Prevalence (ISPM 22)). This model is sufficiently general to be applicable to a variety of insect species; it will be necessary to calibrate and validate it for any given application. Even then, it will only be a supporting tool to assist in making pest management decisions.

For the development of the model, two main situations were considered: (1) the control area is fixed in size (the "fixed-area model") and there is no advancing pest control front, and (2) the control area is expanding according to the "rolling-carpet principle" as described in [5]. Hendrichs et al. [5] describe the basic spatial elements of an AW-IPM program. The first is the core area, in which the aim is to reduce (in case of a suppression strategy) or eliminate (in case of an eradication strategy) the pest species. The core area may contain the actual resource of value, but in other cases, removal of the pest from the core area may simply have a strategic value by protecting crops situated elsewhere or by protecting humans or livestock against disease vectors (in case of a containment or a prevention strategy). The second is a buffer zone that borders the core area on one or more sides and within which control methods attempt to kill the target insects within that zone, including those that enter the zone from outside. The buffer zone is defined as the region of an AW-IPM program that is large enough to prevent the pest insect from moving from outside the buffer to the core area before being destroyed by the control methods operating within the buffer zone. In the case of the fixed-area model, there is a core area to be protected and a buffer zone on all sides of the core area. For the rolling-carpet model, there is a buffer on only one side and pest free zones on the other sides. The width of the buffer zone is central to determining the minimum area of an AW-IPM program, since it defines the smallest possible program.

\section{Methods and Model Development}

2.1. Fixed-Area Model. The fixed-area model considers a rectangular core area, surrounded by a rectangular buffer zone (Figure 1 (left half), Figure 3). This model reflects a situation where the farmer wishes to maintain an area (the core area) pest free or of low pest prevalence without enlarging or moving the area that contains the resource of value. The first aim of the model was to determine the minimum width of the buffer zone given the biological characteristics of the pest and the resources of the AW-IPM 


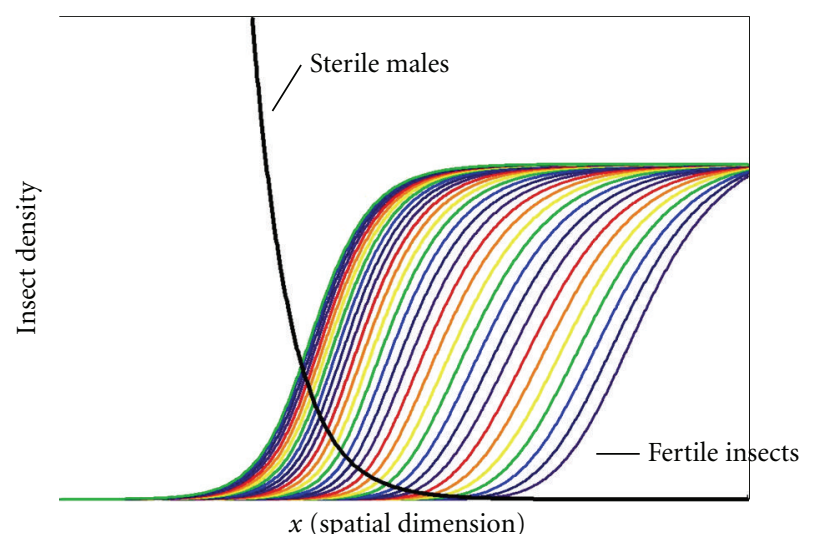

Figure 2: A barrier of sterile male insects theoretically stops a "travelling wave" of fertile insects in a determinable fashion.

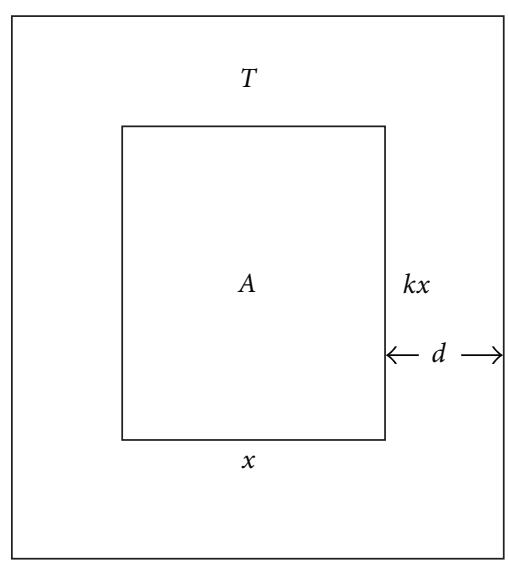

FIgURE 3: Dimensions of the area under control: $A$ is the core area; $T$ is the total rectangle (core + buffer); $x$ is the width of the $A$ area; $k x$ is the length as a multiple of the width; $d$ is the width of the buffer zone $(B)(B=T-A)$.

program. The second aim was to estimate the minimum core area that would result in a viable AW-IPM program.

Numerous simplifying assumptions were made: (i) there is a single target pest insect; (ii) the model does not include the initial process of pest density reduction in the core area because of the difficulties of assessing that aspect, that is, the model assumes that the core area is already a pest free area (or an area of low pest prevalence); (iii) the host density in all areas (the core area, the buffer zone, and outside the buffer zone) was assumed to be at equilibrium; (iv) there is a constant influx of pest insects from the region outside the buffer zone; (v) no artificial movement of the target pest insects by wind, storms, other disturbances, or accidental introduction by humans into the core area occurs.

The rationale for simplifying the model is that managers who aim at managing a pest population using an AW-IPM approach would usually have only limited data on their pest species. A model with a minimal number of parameters (inputs) is, therefore, required if the model is to have a wide applicability. The required parameters will have to be determined for each species before the model can be used since parameter values will vary for different species and environments. The parameters are discussed further below.

The fixed-area model consists of two main components, that is, a biological component (i.e., dispersal) and an economic component (break-even analysis). The dispersal part describes the movement of the insects across the buffer zone and will determine the width of the buffer zone. The economic component of the model will, given a certain width of the buffer zone determined by the dispersal part, allow a calculation of costs and revenues of the control program and will determine the break-even size of the core area at which control costs equal revenues.

2.2. The Rolling-Carpet Model. The same two components, the required width of the buffer zone and the economic requirement of making a profit, apply to the rolling-carpet model, except that the total buffer zone to be supported is located only on one side rather than on all sides around the core area. This might be useful for a pest species confined to a valley and in which the pest insects do not exist at the higher elevations on the sides of the valley.

The rolling-carpet model extends the fixed-area model by introducing a temporal element to the model, that is, the success of the control program permits the core area to be extended regularly when the buffer zone moves onwards. With reference to the scheme shown in Figure 1, the buffer zone will be moved to the right across the control zone to a point where all the area behind the new buffer zone is pest free (or an area of low prevalence is created). This outward movement of the buffer zone will be accompanied by an outward movement of the eradication zone of low prevalence and the population reduction zone. This process could potentially be repeated until an entire pest population has been tackled (this would obviously require sufficient resources to maintain suppression and surveillance activities). This concept was referred to as the rolling-carpet principle [5], since it envisages a gradual movement of the buffer zone across the landscape. In practice, the movement would be more likely to occur in a step-wise manner. The implementation of an AW-IPM program according to the rolling-carpet principle will increase the benefit of the program over time in view of the gradual increase in the size of the core area. The eradication of the New World screwworm Cochliomyia hominivorax Coquerel from Mexico to Panama [6] is a large-scale example of an AW-IPM action program implemented according to this rolling-carpet principle.

The rolling-carpet model does not require any new parameters beyond those required for the fixed-area model, since the essential features of the buffer zone are unaltered by its movement. The only change will be to the economic analysis, since the temporal increase in size of the core area will incur increasing maintenance costs but also increasing benefits.

2.3. Dispersal Models. There are various mathematical approaches for modelling pest dispersal [7]. Regression models, in which insect density following release is regressed 
against distance and time, are relatively easy to develop and solve, and can provide reasonably good predictions. However, they are typically species specific and are not mechanistically linked to insect dispersal behaviour. More mechanistic models of dispersal include metapopulation models, random walks, and diffusion, although metapopulations constitute a special case of random walks, and random walks can yield diffusion equations.

2.3.1. Simple Random Walks. A random walk in one dimension consists of a series of single steps either forwards or backwards along a line. The steps are of length, $\delta$, and the time steps are fixed $(=\tau)$. The final location, $x(n)$, after $n$ steps is the sum of $n$ segments of length $\delta$, some of which are positive and some negative, and as a result the expected position after many steps is the starting position if the probability of going forwards or backwards both equal 0.5. To measure the mean displacement of the final position from the starting position, the square root of the mean of the squares of the final displacements of many random walks is the standard measure used. This corresponds to the standard deviation of the displacements. Since the process of taking a random walk is a binomial event, the resulting distribution of frequencies of $x(n)$ is binomial, and for large $n$ it approaches the normal distribution. The mean square displacement can be written as $E\left(x^{2}(t)\right)=\left(\delta^{2} / \tau\right) t$ and it is convenient to define a diffusion coefficient as $D=\delta^{2} / 2 \tau$ in units of $\mathrm{cm}^{2} / \mathrm{sec}$ [8]. This gives the mean square displacement of $E\left(x^{2}\right)=2 D t$, for $t$ time steps, and the root mean square is $(2 D t)^{1 / 2}$. A consequence of this is that if it requires $t$ time steps to achieve a certain displacement, then it is expected to require $4 t$ time steps to achieve twice the displacement.

If in each time unit, the animal moves in one of the four cardinal directions and if the directions of successive moves are statistically independent, this motion comprises a simple random walk in two dimensions. Each dimension has a mean square displacement of $2 D t$, so in two dimensions the mean square displacement is $4 D t$, and in three dimensions it is $6 D t$.

The diffusion coefficient, $D$, is in units of length squared per unit time and thus is not an intuitive measure of anything of common experience. It is usually estimated by noting the movements of the object under study and tabulating the linear difference between the initial and final positions as well as the number of movements in a given time interval and then computing the means of the squared net distances travelled per unit of time taken over several such random walks. Berg [8] gave an example of a small molecule in water with a diffusion coefficient $D=10^{-5} \mathrm{~cm}^{2} / \mathrm{sec}$. This particle diffuses a distance $x=10^{-4} \mathrm{~cm}$ in $t=x^{2} / 2 D=5 \times 10^{-4} \mathrm{sec}$. It would diffuse one $\mathrm{cm}$ in $t=x^{2} / 2 D=5 \times 10^{-4} \mathrm{sec}$, or about 14 hours. If the distances moved and times for movement are known, then $D$ can be estimated from them by inverting the above procedure. Thus, if an insect takes a random walk and moves a root mean square displacement of $x \mathrm{~cm}$ in $t$ seconds, as a result of $n$ individual movements over a 2dimensional surface, then $D$ can be estimated from these data from $D=x^{2} / 4 t$. Thus, if the $n$ movements yield a root mean square displacement of $24 \mathrm{~cm}$ in 10 seconds, then the estimate of $D$ is

$$
D=\frac{x^{2}}{4 t}=\frac{(24)(24)}{4(10)}=14.4 \mathrm{~cm}^{2} / \mathrm{sec}\left(=0.00144 \mathrm{~m}^{2} / \mathrm{sec}\right)
$$

Because this determination is nonlinear, it is important not to divide $x$ by $t$ before squaring; that is, if the displacement after ten seconds was $24 \mathrm{~cm}$, then the expected displacement after one second would not be $2.4 \mathrm{~cm}$ but rather $2.4(\sqrt{10})=7.59$.

2.3.2. Description of the Diffusion Model. In the limit, as both the length and duration of each move approach 0 , simple random walks become diffusions, described by the partial differential equation [9]

$$
\frac{\partial u}{\partial t}=D \nabla^{2} u
$$

where $\nabla^{2}$ is the Laplacian operator (i.e., the second partial derivatives (of $u$ ) with respect to $x$ and $y: \partial^{2} / \partial x^{2}+\partial^{2} / \partial y^{2}$ in the 2-dimensional case; see also $[10,11])$. For a population of insects released simultaneously at a point, (2) predicts an expanding Gaussian distribution with variance $4 D t$

$$
f(x, y, t)=\frac{1}{4 \pi D t} \exp \left(-\frac{x^{2}+y^{2}}{4 D t}\right) .
$$

Although most insect motion is demonstrably nonrandom, random walk and diffusion equations have been effective at predicting longer-term patterns of insect movement [12-17] because population-level averaging occurs.

A more realistic depiction of insect movement, consists of a series of connected flights in which the distance $(r)$ and direction $(\theta)$ flown are random variables. If the distribution of $\theta$ is uniform, we obtain the model in (2) and (3) above. If the distribution of $\theta$ has a mode at 0 (or some other value; i.e., is not uniform) there will be a tendency to persist in the direction of the previous move, leading to a correlated random walk. In the limit as the $r$ tends to 0 , the process yields an equation known as the telegraph equation

$$
\frac{\partial U}{\partial t}=-\frac{1}{2 \lambda} \frac{\partial^{2} U}{\partial t^{2}}+\frac{v^{2}}{2 \lambda} \nabla^{2} U,
$$

where $v$ is the organism's velocity and $\lambda$ is its rate of change of direction. For large elapsed times, the predictions of (2) and (4) converge, differing by less than $5 \%$ for $t>10.5 / \lambda$. Both models predict similar rates of spread for large values of $t$. Holmes [18] compared predictions for diffusion and telegraph equations for a variety of organisms, including the cabbage butterfly (Pieris rapae (L.)), gypsy moth (Lymantria dispar (L.)), European starling (Sturnus vulgaris (L.)), collared turtledove (Streptopelia decaocto Frivaldszky), and black death (Yersinia pestis (Lehmann and Neumann) van Loghem), and predictions for the two models differed by $<8 \%$. Similar convergence between the predictions of (2) and other correlated random walk formulations were reported by Kareiva and Shigesada [19]. Diffusion models thus provide robust predictions of animal dispersal patterns. Here, we describe the situation for a single buffer separating a pest population from a protected area. 
2.4. The Biological Component: Width of the Buffer Zone. The pest population will have a certain ambient density outside of the buffer zone and will disperse from outside into the buffer zone. Because control measures are imposed within the buffer zone, the density of the pest will decrease from the outer edge of the buffer to the inner edge. The width of any buffer zone around a core area should be large enough to bring the density of the pest to zero (in case of a pest free area) or close to zero (in the case of an area of low pest prevalence) in the core area $(A)$ (Figure 1). The buffer zone should, therefore, be wide enough to prevent a gravid female insect and any of its offspring crossing the buffer zone.

If the population is growing and dying, as well as diffusing, then an appropriate model would be

$$
\frac{\partial F(x, t)}{\partial t}=D \nabla^{2} F(x, t)+g F(x, t)
$$

where $g$ is the growth function. If $g$ is linear and births and deaths can be separated, then

$$
\frac{\partial F(x, t)}{\partial t}=D \nabla^{2} F(x, t)+\beta F(x, t)-\delta F(x, t),
$$

in which $\beta F(x, t)$ and $\delta F(x, t)$ are the instantaneous birth and death rates. We want boundary conditions such that at the outside of the buffer zone, $F(0, t)=F_{0}$, where $F_{0}$ is the density of insects at the outer edge of the buffer as a result of the influx of insects, and at the inside edge of the buffer, $F(w, t)=a$ small proportion of $F_{0}\left(\right.$ e.g., $\left.10^{-6}\right)$, so that almost all the insects have been killed before reaching the other side of the buffer (of width $w$ ).

If we are manipulating the death rate within the buffer by traps that are evenly spread out to cover the whole of the buffer region, then $(\beta F-\delta F)$ will be negative, because now $\delta$ consists of the sum of natural and imposed mortality from traps or any other control source. To simplify the treatment here, we assume that we are dealing with a steady-state situation in which the insects have been diffusing and the buffer has been under control for a long time. In that case, the time derivative is zero, since nothing is changing over time; only the space derivative is still non-zero. This yields the steady-state equation

$$
D \nabla^{2} F=(\delta-\beta) F
$$

and this has solutions proportional to $e^{-\gamma x}$, where $\gamma^{2}=$ $(\delta-\beta) / D$ [20]. Assuming $F(x)=c e^{-\gamma x}$, the boundary conditions dictate that $c=F_{0}$ and that $F_{0} e^{-\gamma w}=10^{-6} F_{0}$. Taking logarithms, $-\gamma w=\ln \left(10^{-6}\right)=-13.8$. This leads to the minimum buffer width

$$
w=\frac{13.8}{\gamma}=\frac{13.8}{[(\delta-\beta) / D]^{1 / 2}} .
$$

In this case, the diffusion coefficient is determined in the same way as it was for random walks. If a decrease down to $10^{-6}$ of the original density outside the buffer $\left(F_{0}\right)$ is not satisfactory, then another small fraction can be chosen and the 13.8 will be something else. The units of $w$ in (8) are in the units of $D$, and the units of $\beta$ and $\delta$ must be the same as those of $D$. Thus, if the units of $\beta$ and $\delta$ are in terms of numbers per week, then $D$ should also be in terms of distance ${ }^{2}$ per week.

If sterile insects are to be used as the control method, then the simplest case to solve is the case in which the release of sterile insects is proportional to the ambient population, in which case $\beta$ is to be manipulated [21], rather than $\delta$, and the development is similar. This case has the decrease in fertility being constant because the sterile release rate is proportional to the wild population, and thus the fertility (or sterility) ratio is constant. If this ratio can be determined to be some constant, $\beta^{\prime}$, then it will have to be small enough that again $\delta>\beta^{\prime}$ and then the determination of minimum buffer width proceeds in the same way as above, with $\beta^{\prime}$ replacing $\beta$ and $\delta$ only consisting of natural mortality in (8) above. The rest of the calculations are identical to those in (6) to (8).

If sterile releases are to be maintained at a constant level throughout the buffer region, then dropping $x$ and $t$, the equations will take the form [22]:

$$
\begin{gathered}
\frac{\partial F}{\partial t}=D \frac{\partial^{2} F}{\partial x^{2}}+\beta F \frac{F}{F+S}-\delta F, \\
\frac{\partial S}{\partial t}=D \frac{\partial^{2} S}{\partial x^{2}}+R(x)-\delta S,
\end{gathered}
$$

where $F$ and $S$ are the densities of fertile and sterile insects, $\delta$ is the rate of natural mortality of fertile or sterile insects, and $R(x)$ is the rate of sterile fly release. This model appears intractable analytically, and would have to be solved numerically for each particular situation. In the steadystate case, the partial differential equations become ordinary differential equations.

Equation (9) predict that sterile density rapidly achieves a time-independent steady state under continuous release (the sterile male curve in Figure 2). For many functional forms $g(F, S),(5)$ predicts that the fertile pest insect population will propagate into a pest free area as a travelling wave. Below a threshold release rate of sterile insects, these travelling waves can propagate through the area under SIT. For sterile release rates above this threshold, the travelling waves of expansion of the fertile pest population stall upon encountering the sterile barrier of the buffer. The fertile curves in Figure 2 depict the travelling wave at fixed time intervals (see also [23]). As the wave nears the sterile insect buffer, the curves plot ever closer together (finally plotting on top of one another) indicating that the wave has stalled. The inflection point of the stalled wave is a useful benchmark of the penetration of the pest population into the protective buffer zone. Within the buffer zone, the density of fertile pest insects typically decays nearly exponentially from the inflection point at a rate $\sqrt{\delta_{f} / D_{f}}$, where $\delta_{f}$ is the death rate of the fertile insects and $D_{f}$ is the diffusion coefficient of the fertile insects. Accurate densities can be calculated numerically using the parameters above.

2.5. Parameters for Inclusion in the Diffusion Model. A minimum set of parameters was identified for inclusion in the diffusion model (Table 1). If SIT is a component 
TABLE 1: Biological dispersal model parameters used in the models to estimate a minimum area for an area-wide IPM program, including the sterile insect technique.

\begin{tabular}{|c|c|c|c|}
\hline Parameter $^{\mathrm{a}}$ & Units & Description & Notes \\
\hline$\beta$ & $\mathrm{day}^{-1 \mathrm{~b}}$ & Birth rate & $\begin{array}{l}\text { May vary between } \\
\text { fertile and sterile insects }\end{array}$ \\
\hline$\delta$ & day $^{-1}$ & Death rate & $\begin{array}{l}\text { May vary between } \\
\text { fertile and sterile insects }\end{array}$ \\
\hline$\sigma$ & - & $\begin{array}{l}\text { Sterile male } \\
\text { competitiveness }\end{array}$ & $\begin{array}{l}\text { Dimensionless. Ranges } \\
\text { from } 0 \text { to } 1\end{array}$ \\
\hline$D$ & $\mathrm{~km}^{2} \mathrm{day}^{-1}$ & $\begin{array}{l}\text { Diffusion } \\
\text { coefficient }\end{array}$ & $\begin{array}{l}\text { Scales the rate of } \\
\text { population spread. May } \\
\text { vary between fertile and } \\
\text { sterile insects }\end{array}$ \\
\hline$R$ & $\begin{array}{c}\text { ind }^{\mathrm{c}} \\
\mathrm{km}^{-2} \mathrm{day}^{-1}\end{array}$ & $\begin{array}{l}\text { Sterile release } \\
\text { rate }\end{array}$ & \\
\hline$A$ & ind $\mathrm{km}^{-2}$ & $\begin{array}{l}\text { Ambient } \\
\text { population } \\
\text { density }\end{array}$ & $\begin{array}{l}\text { Used to estimate density } \\
\text { dependence parameters }\end{array}$ \\
\hline
\end{tabular}

of the strategic approach, the parameters are the diffusion coefficient, daily birth and death rates, ambient density of the fertile population, competitive ability of the released sterile insects, and sterile release rate as a multiple of the ambient density of fertile insects. Three basic modes of insect dispersal were identified (active, wind borne, and human), but only active dispersal was included in the basic diffusion model. The other modes were considered but, in view of their complex and unpredictable nature, were not included.

2.6. An Excel Model to Approximate the Diffusion Model. An alternative approach to modelling the minimum width of the buffer zone is the use of direct stochastic simulation. Whereas the diffusion equation approach runs until the advancing wavefront is halted by the sterile releases, that is, it reaches steady state, a simulation need not run until steady state (which may take an unrealistically long time in relation to seasonal variation). Such a simulation model has been written (by SG, and available from him on request) using Excel VBA. The simulation runs as a macro in Excel and allows the user to vary most of the relevant biological parameters. Rather than run to steady state, the simulation ends after a user-defined number of days (typically a year or more).

As with the diffusion equations, the simulation model uses a normal distribution of movement lengths. Instead of solving the diffusion equations, the simulation tracks the movements of thousands of individuals (representing both wild and sterile flies) on a grid consisting of 20000 lattice squares, each $20 \mathrm{~m}$ by $20 \mathrm{~m}$. Sterile individuals are released at user-determined points in the middle portion of the lattice (to simulate the buffer zone). Initially, wild flies are found only on one side of the buffer (the infested area) and as the simulation proceeds, the daily distribution of wilds and steriles is graphed. At the end of the simulation, the penetration benchmark is recorded as the density of wild flies in the production area. The simulation uses a similar parameter set to that used in the diffusion equations (Table 1), but allows all the parameters to be varied by the user. The simulation uses a Leslie matrix projection [24] to establish a stable age distribution for the wild population. The simulation also includes an age-related accelerating death rate (Gompertz function). The diffusion coefficient, $D$, of the simulation can be calculated from the observed positions of flies as they spread from the sterile point releases. By adjusting the variance of the daily dispersal distance used in the simulation, the simulated $D$ can be set to match the $D$ used in the diffusion equation models.

\subsection{The Economic Component: Size of the Core Area}

2.7.1. First Approach: Variable Width and Length of the Core Area. The size of the buffer zone $(B)$ is determined by biological parameters related to dispersal ability of the pest (Figure 3 ). For the core area $(A)$, an economic approach was adopted for assessing its minimum size depending on the size of the buffer zone, as determined by the diffusion model. This is because there seems to be no obvious biological constraints on the size of the core area whereas there will be obvious economic constraints resulting from the costs incurred in maintaining the protective buffer zone. The size of the core area can be calculated resulting from the "breakeven point" (if it exists) at which the revenues equal the costs. For any larger core area, the revenues obtained will exceed the costs of maintaining the core area and buffer zone, including control costs, surveillance, and quarantine, resulting in a net profit.

In a basic model, a rectangular protected area $(A)$ is assumed with minimal assumptions (Figure 3). The strategy here is to compute cost and revenue curves for core areas and buffer zones of different sizes and note where they intersect (i.e., the break-even point).

Costs of the Control and Associated Efforts in Relation to Size of the Buffer and Core Area. The area of the smaller rectangle $(A)$, core area, is

$$
A=k x^{2}
$$

where $x$ is the width of the inner rectangle, $k x$ is the length, and $k$ is the ratio of length to width of the inner rectangle. The area of the larger $(T)$ rectangle is

$$
T=k x^{2}+2 d x(k+1)+4 d^{2},
$$

where $d$ is the distance between the inner and outer rectangles. The area of the buffer zone $(B)$ equals $T-A$ and is

$$
B=T-A=2 d x(k+1)+4 d^{2} .
$$

The cost of control in the buffer is

$$
\left[2 d x(k+1)+4 d^{2}\right] q,
$$


where $q$ is the cost of control and associated activities per unit area. The cost of control in the core area $(A)$ is

$$
k x^{2} w q
$$

where $w$ is the reduction in effort within $A$ due to there being fewer, or no, pests there. The cost of additional controls and associated activities such as surveillance, quarantine, sanitation, and staffing are contained within $w$.

Each of these costs is assumed to be a fixed cost per hectare. Emergency response programs, including extra surveillance have not been included. The total cost is a quadratic function of $x$

$$
T_{c}=\left\{\left[2 d x(k+1)+4 d^{2}\right] q\right\}+\left\{k x^{2} w q\right\} .
$$

Revenue of the Resource of Value. The revenue of the resource of value will be related to the area in the following way. The revenue of the core area $=[$ value per unit area $] \times[$ size of the protected area $]=(v)\left(k x^{2}\right)$, where $v$ is the value per unit area.

The revenue of the buffer zone $=e v\left[2 d x(k+1)+4 d^{2}\right]$, where, $e v$ is the reduced value of goods from the buffer zone. The benefit of the buffer zone is expected to be zero or low as commercial production may not exist or production is small and targeted to the core area. Thus, $e$ takes on values between zero and one. The total revenue is thus

$$
T_{b}=e v\left[2 d x(k+1)+4 d^{2}\right]+v k x^{2} .
$$

Break-Even Point. The break-even point can be calculated from the intercept of cost and benefit curves from above. Cost $=$ Revenues when

$$
\begin{aligned}
& \left\{\left[2 d x(k+1)+4 d^{2}\right] q\right\}+\left\{k x^{2} w q\right\} \\
& =v k x^{2}+e v\left[2 d x(k+1)+4 d^{2}\right] .
\end{aligned}
$$

Widths of the Core Area and the Buffer Zone. In order to make a profit, the following quadratic inequality must be satisfied

$$
P=k(v-q w) x^{2}+2 d(k+1)(e v-q) x+4 d^{2}(e v-q)>0 .
$$

Costs: $q$ cost per unit area for buffer zone $B$,

$w$ lower cost per unit area for core area $A$.

Revenues: $v$ value of resource per unit area for core area $A$, $e$ lower value per unit area for buffer zone $B$.

Profit: $P$ equals revenues minus cost.

Four cases have been identified involving $v, q, w$, and $e$ based on inequality (14) above (Table 2). If $v>q w$ and $e v>$ $q$, then all terms will be positive and the inequality is always satisfied ( $k, d$, and $x$ must be positive to be realistic) and thus any size of core area will be profitable. On the other hand, if $v<q w$ and $e v<q$, then all terms will be negative, and the inequality can never be satisfied, and thus there is no size of core area that is profitable. If $v>q w$ and $e v<q$, then the term in $x^{2}$ will eventually dominate if $x$ becomes large
TABLE 2: Inequalities between factors determining the profitability of a control program. The letters stipulate the following: $q$ : cost per unit area for the buffer zone, $B$; $w$ : cost per unit area for the core area, $A$, as a proportion of $q ; v$ : value of the resource per unit area for the core area, $A ; e$ : value of the resource per unit area for the buffer, $B$, as a proportion of $v$.

\begin{tabular}{ll}
\hline$v>q w$ and $e v>q$ & $v>q w$ and $e v<q$ \\
\hline Always profitable & Break-even exists \\
$\begin{array}{l}\text { Parabola upright; roots negative } \\
\text { or complex }\end{array}$ & $\begin{array}{l}\text { Parabola upright; one positive } \\
\text { root }\end{array}$ \\
\hline$v<q w$ and $e v>q$ & $v<q w$ and $e v<q$ \\
$\begin{array}{l}\text { Break-even exists } \\
\text { Parabola inverted; one positive } \\
\text { root }\end{array}$ & $\begin{array}{l}\text { Pever profitable } \\
\text { Parabola inverted; no positive }\end{array}$ \\
\hline
\end{tabular}

enough, and there is thus a break-even point and the core area can be profitable above this size. If $v<q w$ and $e v>q$, then the inequality is satisfied for small values of $x$, and may also be satisfied for large values, depending on the relative sizes of $(v-q w)$ and $(e v-q)$.

These four inequalities determine whether or not a break-even point exists or whether there is no size of the core area that is unprofitable (upper left of Table 2) or profitable (lower right). One can maximize the profit function in inequality (18) by differentiating with respect to $x$. We get that profit is maximized when

$$
x=\frac{d(k+1)(q-e v)}{k(v-w q)}
$$

which is only positive in the two cases in Table 2 where a break-even point exists.

It is worthwhile to ask if there is a value of $k$ that will maximize profit. Inequality (18) indicates that profit is a linear function of $k$. Thus, any profitable set of parameter values (i.e., $v>q w$ and $e v>q$ ) will be made more profitable by increasing $k$, and any unprofitable values (i.e., $v<q w$ and $e v<q$ ) will be made more unprofitable by increasing $k$ (if $x$ is held fixed). The case where $v>q w$ and $e v<q$ will be unprofitable for small $k$, but once $k$ increases past the break-even point, higher values of $k$ will increase profit and no optimum exists. The last case, where $v<q w$ and $e v>q$, is unrealistic, as here the buffer zone is profitable, but the core area is not, so that small values of $k$ will yield a profit, but larger ones will not; again no optimum exists, except for $k=0$.

If the size of the core area is kept at a fixed value, then it is easy to show that a value of $k=1$ maximizes profit. This is done by substituting $x / k$ for the width of the core area, and leaving the length at $k x$, so that the area remains at $x^{2}$ for all $k>0$. One then computes a new profit function and differentiates it with respect to $k$ and finds that the derivative is zero at $k=1$.

2.7.2. Second Approach: Predetermined Width of the Core Area. Another approach to the problem of finding the minimum area for an AW-IPM program to be feasible would be by 
predetermining the width of the core area, rather than the shape of it. The core area might be in a valley where the width is constrained by the width of the valley, and thus in the economic model, one could predetermine $x$ and then solve for $k$, rather than the other way around. Similar results were obtained with four separate cases. The four cases appear the same as those found before, but the intermediate cases are not identical, as the size of the buffer zone is not the same here when $k$ goes to zero, because the width remains at $x$ whereas the width went to zero in the previous treatment as the size of the core area decreases. This approach also results in (18) for the break-even point, and inequality (19). If we then solve for $k$

$$
k>\frac{\left(2 d x+4 d^{2}\right)(q-e v)}{\left[x^{2}(v-w q)-2 d x(q-e v)\right]} .
$$

This also yields four cases, two of which involve no minimum size for the core area, being profitable for all areas or unprofitable for all areas. The other two involve either the buffer zone being profitable and the core area unprofitable (which is unrealistic) or the buffer zone being unprofitable and the core area profitable.

If $q>e v$, so the buffer zone is not profitable, then in order for the system as a whole to be profitable, we need

$$
x>\frac{2 d(q-e v)}{(v-w q)},
$$

and then the value of $k$ is found from the equation above. Thus, the width of the core area needs to be a certain multiple of the width of the buffer zone in order for any value of $k$ to yield profitability.

These two approaches are compatible in the sense that they yield the same minimum areas but by different methods. If one chooses $k$ and then finds $x$ by the first method, one gets a certain value of $x$. If one substitutes this value of $x$ into the equation and solves for $k$ using the second method, then one calculates the value of $k$ that was used the first time. However, they will not, in general, yield the same results because of the differing constraints used. In the first method, the shape of the core area is predetermined by choosing $k$; in the second method, the shape is calculated by choosing $x$, and will not usually be the same as that chosen for the first method. A numerical example will illustrate this.

Use parameter values as follows (the choice is somewhat arbitrary): $d=1.0, q=10.0, w=0.2, v=20.0, e=0.1$. Choosing $k=1.6$, we calculate $x$ for the break-even point by the first method to be 2.0. Choosing $x=2.0$, we calculate $k$ by the second method to be 1.6. Thus, the two methods are consistent. However, if we had chosen $x$ to be 3.0, then using the second method we would find that $k=0.70$. Thus, the two methods may produce different (but still compatible) results.

This second approach to the economic model resulting in inequality (15) is more suited to the rolling-carpet scenario, as the width $(x)$ is fixed and the length (determined by $k$ ) will vary. The solution of (14) for $k$ will give the break-even point, above which the core area plus buffer zone can show a profit. Unless logistical constraints pose a limit, this value of $k$ is perhaps the one to plan on achieving when setting up the rolling-carpet scenario. The difference here is that the buffer zone is only on one side (the front), as the other sides are assumed to be pest-free. As shown in Figure 1, however, there are three zones outside the core area in which control is imposed, and thus the total buffer zone may be comparable to that of the fixed-area model.

\section{Case Studies}

3.1. Tsetse Flies. Yu et al. [25] simulated the dispersal of tsetse flies using a 2-dimensional random walk model. In this model, both distance moved and direction of movement were random variables. Hourly distances moved were taken from Dean et al. [26], varying between 0.8 and $4.08 \mathrm{~m}$. The mean distance moved per week was taken from data of Jackson [27] as $334 \mathrm{~m}$, and hourly distances were adjusted accordingly, as the data from Jackson were judged to be better than those of Dean et al. The resulting hourly distances were fitted to a Weibull distribution. Yu et al. [25] assumed that both birth and death rates were random variables and that the death rate was determined by both natural mortality and one of three control measures tested, being (i) insecticide application, (ii) vegetation clearing and wild animal depopulation, and (iii) the use of tsetse traps. Birth rates varied between 0.0 and 0.1 per day. Ninetynine percent of adult tsetse died by 84 days of age. The 99\% confidence upper bound for the lifetime straight-line distance travelled was calculated to be $1.71 \mathrm{~km}$. Only a small proportion of them would have travelled in any given direction. They simulated the movement of flies into an uninhabited area. To do this they assumed that 5110 adult tsetse were uniformly distributed over an area of $100 \mathrm{~km}^{2}$ and were moving randomly as indicated above. Their simulations indicated that the upper $99 \%$ confidence limit for movement was $18.7 \mathrm{~km}$ by the year 10 . They then considered two adjacent areas, one tsetse-infested and the other tsetse-free. To prevent tsetse from moving from the infested area to the noninfested area, a protective buffer was simulated, and two measures were considered: the width, $w$, of the buffer, and the probability of a fly successfully crossing into the noninfested area. The probabilities of successful crossing were in the order of $1 \%$ for a buffer width of $1000 \mathrm{~m}$ using any of the three control methods. When control methods were combined, these probabilities were reduced. However, since the lifetime displacement was determined to be $1.71 \mathrm{~km}$, the buffer would likely need to be at least that wide. In fact, the buffer widths would have to be increased considerably to guarantee that less than one insect successfully invaded the tsetse-free area.

If sterile releases (SIT) were to be used in the buffer zone, the birth rate in Yu et al.'s model would have to be modified according to the usual sterility function [28], which would introduce a nonlinearity into the birth rate and complicate the computations. This could be circumvented by assuming that the sterile release rate would decrease as the population density decreased throughout the buffer zone; this would be simpler to compute (21) and gives a conservative estimate of the buffer width (i.e., an overestimate), as the latter 
use of SIT is less efficient than the release of constant numbers. Alternatively, the standard approach to SIT could be calculated numerically.

We have only dealt with the dispersal model and buffer here, as the economic component is fully explained in the section on the Mediterranean fruit fly. Also, the parameter values used here were for illustrating the procedure and should not be assumed to be realistic in any real control program.

\subsection{The Mediterranean Fruit Fly. The Mediterranean fruit} fly, Ceratitis capitata (Wiedemann), was chosen as an example pest because numerous AW-IPM programs that include the SIT have successfully targeted this species. These provide some practical experience against which to assess the model outputs. In addition, the Mediterranean fruit fly is relatively well studied in terms of its biology [29, 30], mobility and dispersal [31-35], ecology [36, 37], and so forth. The parameter values assumed here may vary with location and are presented only to illustrate the procedure.

Background information is as follows. (a) Insect density-4000 fertile insects/ha at peak; (b) birth rate-six-fold increase per generation; (c) mortality-10\% per day for adults; (d) adult lifespan-ten days; (e) time from egg to adult-about 30 days in tropical and subtropical conditions and 45 days in temperate regions; (f) dispersal ratemaximum of $1.5 \mathrm{~km}$ per lifetime. However, $D$ (the diffusion coefficient) used in the diffusion model is measured in terms of $\mathrm{km}^{2} /$ day, which has no simple biological meaning. The dispersal parameter, $D$, is obtained from $D=1.5^{2} / 8(l)$, which in this case is $2.25 / 8(10)=0.028$. This leads to a minimum buffer width of about two $\mathrm{km}$; (g) core area as a pest free area or an area of low prevalence (the target pest density is potentially different); (h) assumed coststhe cost of the SIT package (production, packing, and transport to the releases site is $\$ 500 /$ million sterile insects) for the eradication phase is about $\$ 130$ per ha per year assuming a sterile fly density of 5000 males per hectare and weekly releases throughout the year amounting to 52 releases ( $+30 \%$ for other eradication and surveillance measures). The cost is substantially reduced during the fly-free phase or maintenance phase (to ca $1 / 5$ of the cost during the eradication phase) since only surveillance and quarantine activities are conducted to maintain the fly-free status. The cost of insecticide-bait treatments for initial suppression prior to the release of sterile flies is about US $\$ 120$ per ha per year assuming a cost of $\$ 12$ per treatment per hectare and 10 treatments per year. (i) Assumed revenue- $\$ 5000 /$ ha (at 10 tonnes/ha and $\$ 500$ per tonne, which is an average of many fruit commodities) in the core area $A$ and $\$ 0$ in the buffer zone.

Recent studies of medfly dispersal suggest that a $2 \mathrm{~km}$ buffer zone is a reasonable starting point for the models presented here. Meats and Smallridge [35] studied dispersal of medfly across a grid of 3750 surveillance traps at distances up to $10 \mathrm{~km}$. They found that $90 \%$ of released flies remained within $0.4-0.7 \mathrm{~km}$ of the release point. Their results were consistent with a number of earlier studies that had investigated dispersal over shorter distances (up to $0.7 \mathrm{~km}$; references in Meats and Smallridge [35]). Furthermore, Wong et al. [32] found little difference in dispersal of wild and irradiated medfly. Therefore, most wild medfly entering a $2 \mathrm{~km}$ buffer zone will die before crossing the zone, and any offspring produced in transit should suffer lowered fertility due to matings with the overflooding sterile flies. The actual number of wild flies crossing the buffer zone will then depend on the details of density, lifespan, fertility, and movement of the wild flies. The dispersal model will provide a calculated value of the dispersal coefficient $D$, which will, in turn, determine the calculated width, $d$, of the buffer zone, $B$.

The economic analysis will provide the calculated size of the core area $A$. The Excel worksheet referred to earlier was parameterized for the Mediterranean fruit fly, although it would be suitable for other species simply by using appropriately different parameter values. The proportions of flies, being released at a source, that are expected to disperse to various distances in one day are tallied, based on the Excel worksheet. When run for one year, it predicted that two $\mathrm{km}$ would seem to be a reasonable buffer width based on the parameter values assumed here.

Using the values given above, we can assign values to the parameters in the economic model as follows. Assume that a square core area is desired, so that $k=1$; also, $d=2$. Costs per unit area for the buffer $(q)$ are $\$ 130 / \mathrm{ha} / \mathrm{yr}$ $+30 \%+\$ 120 / \mathrm{ha} / \mathrm{yr}=\$ 28900 / \mathrm{km}^{2}$. Costs for the core, $w$, are zero. Benefits, $v$, in the core area are $\$ 5000 /$ ha/ $/ \mathrm{yr}=$ $\$ 500000 / \mathrm{km}^{2} / \mathrm{yr}$. Benefits from the buffer, $e$, are zero. The quadratic relationship between the width of the core area $A$ and the net profit (inequality (14)) is then (500000) $x^{2}+$ $2(2)(2)(-28900)+4(4)(-28900)=0$ for the breakpoint, which gives a break-even point at a width of $1.2 \mathrm{~km}$ of the core area $A$ for a biological buffer distance of $2 \mathrm{~km}$. The units of $x$ and $d$ must be the same. This conforms to the upper right case in Table 2 .

\section{Discussion}

It will be important to consider a risk of incursions into the core area, which result in pest density rising above the chosen threshold density in the core area $A$. The latter depends on the strategy chosen, that is, if the aim of the program is to establish a pest free area then the threshold density is zero, but if the core area is aimed to be an area of low pest prevalence, the chosen threshold density could be the agreed threshold between the exporting and importing country.

There are several factors which may contribute to incursions into the core area: inadequate width of the buffer zone, high dispersal rate of the pest insect, human introductions, wind-borne incursions, varying control efficiency, failure in the execution of the operation, and size of the core area $A$.

There is a distinct relationship between the risk of introductions and the size of the protected (core) area. The risk of human introductions increases with the size of the core area $A$, since the probability of an introduction into a large area is greater than that into a small area simply based on assumed random movement of humans. It is recognized that human activity is not random, but a larger area would likely be used by more humans than a small area. Similarly, 
the risk of wind-borne incursions would increase with the size of the core area $A$ for the same reason as for humans.

If a large core area is partitioned into a set of smaller subareas, then the edge subareas should be more vulnerable to incursions than the centre. This suggests the need for large core $(A)$ zones and increased surveillance near the perimeter of the core area $A$.

The diffusion approach is useful for relatively homogeneous buffer areas with no obstacles to dispersal or other factors that interfere with insect movement. If such obstacles do exist, then the use of the diffusion equations becomes less precise. Also, if there are prevailing winds that affect insect movement, they will have to be factored into the calculations of buffer width. A modification of the simple diffusion equations that would accommodate this is to use a diffusion equation with a term for drift, the so-called Fokker-Planck equation (14), which is somewhat more complicated than the simple diffusion equation with growth. The application of diffusion to the tsetse case gave an estimate of between one and two $\mathrm{km}$ width for the buffer zone. There appears to be little agreement on how far tsetse can disperse, and the estimates from different species and among investigators vary considerably. For example, Brightwell et al. [38] reported $175 \mathrm{~m} /$ day as the root mean square displacement. Yu et al. [25] gave $1.71 \mathrm{~km}$ as the lifetime straight-line distance travelled for tsetse. Challier [39] reported the average daily displacement to be $252 \mathrm{~m}$. Glasgow [40] reported the displacement after release to be $700 \mathrm{~m}$ in the first week and then $200 \mathrm{~m}$ each week thereafter. With such a variety of figures to choose from, the derivation of any definitive buffer width is elusive.

The economic model parameterized for the Mediterranean fruit fly was used to demonstrate the relationship between key variables. It is essential to recognize the limitations of the approach that has been taken here, and especially to use the model output values with caution. Any improved model will still suffer from the inherent limitations of its assumptions, but the preliminary nature of the current model means that special care is needed in this area. The approach taken was to develop parameter estimates that produced a break-even value for the project.

The economic analysis can be easily produced using a spreadsheet, allowing much faster calculation than the diffusion models. Therefore, it is suggested that the following general process could be used in the decision-making process.

(1) Obtain an estimate of the maximum dispersal range of the pest. This provides an initial value for the width of the buffer zone.

(2) The economic spreadsheet analysis can then be performed to test the financial viability of the project, using the approximate estimate of the size of the buffer zone. The economic analysis will provide an estimate of the minimum size of the core area required to support the project.

(3) The diffusion model can then be run (using a minimum core area estimate).

This process is likely to be the most efficient method of combining the two elements of the model.
For the reasons outlined above, the model was kept as simple as possible. However, various important complexities could be added to future, more complex versions of the model. Habitat heterogeneity can affect the dispersal rate of an insect, which here has been assumed to be a constant. Also, clumping of the pest distribution may affect the efficiency of control. Wind and rainfall can increase dispersal rates in insects, and, therefore, can increase the potential introduction of the pest into a pest free area, although proper surveillance systems could address these rare events, and an appropriate response could be developed using additional control. Directed dispersal by insects occurs within the buffer zone but may take place in all directions in relation to wind. For example, cross-wind or upwind dispersal may be used by insects to detect mates or habitat. Downwind dispersal may occur outside the boundary layer and is difficult to predict. Finally, seasonal temperature differences also may have a major effect on pest activity, survival, and efficiency of the sampling device.

\section{Conclusions}

The preliminary testing of the conceptual model suggests that it should be possible to estimate the minimum area required for an AW-IPM program, with or without an SIT component, to be technically feasible and economically justifiable. Calculations using the assigned parameter values suggested that for the Mediterranean fruit fly, the minimum buffer width could be as small as $2 \mathrm{~km}$ (surrounding a core production area of at least $43 \mathrm{ha}$ ). These results were in general accord with expectations based on experience of past AW-IPM programs that included the release of sterile insects. As expected, high ambient pest densities (i.e., untreated tropical and subtropical populations of Mediterranean fruit fly inhabiting vast host areas like coffee plantations) rendered SIT ineffective over much larger distances.

A possible limitation arises since the diffusion models employed are equilibrium models. In temperate regions, seasonality exerts a major effect on pest survival and activity. While seasonal temperature effects could be included in the model via variable birth and death parameters, the effect on the speed at which the model approaches equilibrium has not been determined. If it seems likely that seasonality will prevent the model from reaching equilibrium, then the user will have to be careful to only use the early generations of the model in assessing the size of the buffer zone.

The portability of the model between organisms will need to take account of the differences in values for key parameters, the simplistic depiction of the pest biology and the applicability of the assumptions to each species. Any generic model will have limitations for certain insect groups. Important complexities include inherited sterility (Lepidoptera treated at lower doses), aggregation behavior (some Coleoptera), or complex biology.

\section{Acknowledgments}

The authors acknowledge support from the New Zealand Foundation for Research Science and Technology investment 
in "Better Border Biosecurity" for the contribution of D.M. Suckling towards the paper. They thank Pauline van den Driessche for checking their development of (5) to (8). This paper is the result of a consultants meeting that was organised and funded by the Joint FAO/IAEA Division of Nuclear Techniques in Food and Agriculture.

\section{References}

[1] National IPM Network, 2001, http://www.ag.ndsu.nodak.edu/ aginfo/ndipm/ipmdefinition.htm.

[2] J. Hendrichs, A. S. Robinson, P. Kenmore, and M. J. B. Vreysen, "Integrated area-wide pest management: principles and prospects," in Area-Wide Control of Insect Pests: From Research to Field Implementation, M. J. B. Vreysen, A. S. Robinson, and J. Hendrichs, Eds., Springer, Dordrecht, The Netherlands, 2007.

[3] W. Klassen, "Area-wide integrated pest management and the sterile insect technique," in Sterile Insect Technique. Principles and Practice in Area-Wide Integrated Pest Management, V. A. Dyck, J. Hendrichs, and A. S. Robinson, Eds., pp. 39-68, Springer, Dordrecht, The Netherlands, 2005.

[4] V. A. Dyck, J. Hendrichs, and A. S. Robinson, Eds., Sterile Insect Technique. Principles and Practice in Area-Wide Integrated Pest Management, Springer, Dordrecht, The Netherlands, 2005.

[5] J. Hendrichs, M. J. B. Vreysen, W. R. Enkerlin, and J. P. Cayol, "Strategic options in using sterile insects for area-wide integrated pest management," in Sterile Insect Technique. Principles and Practice in Area-Wide Integrated Pest Management, V. A. Dyck, J. Hendrichs, and A. S. Robinson, Eds., pp. 563-600, Springer, Dordrecht, The Netherlands, 2005.

[6] J. H. Wyss, "Screw-worm eradication in the Americasoverview," in Proceedings of the Area-Wide Control of Fruit Flies and Other Insect Pests. International Conference on Area-Wide Control of Insect Pests, and the 5th International Symposium on Fruit Flies of Economic Importance, Penerbit Universiti Sains Malaysia, Penang, Malaysia, May 1998.

[7] R. E. Stinner, C. S. Barfield, J. L. Stimac, and L. Dohse, "Dispersal and movement of insect pests," Annual Review of Entomology, vol. 28, pp. 319-335, 1983.

[8] H. C. Berg, Random Walks in Biology, Princeton University Press, Princeton, NJ, USA, 1983.

[9] E. C. Pielou, An Introduction to Mathematical Ecology, John Wiley \& Sons, New York, NY, USA, 1969.

[10] W. G. Rudd and R. W. Gandour, "Diffusion model for insect dispersal," Journal of Economic Entomology, vol. 78, no. 2, pp. 295-301, 1985.

[11] L. Edelstein-Keshet, Mathematical Models in Biology, Random House, New York, NY, USA, 1988.

[12] T. Dobzhansky and S. Wright, "Genetics of natural populations. X. Dispersal rates in Drosophila pseudoobscura," Genetics, vol. 82, no. 3, pp. 493-506, 1976.

[13] P. Turchin, Quantitative Analysis of Movement: Measuring and Modeling Population Redistribution in Animals and Plants, Sinauer Associates, New York, NY, USA, 1998.

[14] J. G. Skellam, "Random dispersal in theoretical populations," Biometrika, vol. 38, no. 1-2, pp. 196-218, 1951.

[15] A. Okubo, Diffusion and Ecological Problems: Mathematical Models, Springer, New York, NY, USA, 1980.

[16] P. M. Kareiva, "Local movement in herbivorous insects: applying a passive diffusion model to mark-recapture field experiments," Oecologia, vol. 57, no. 3, pp. 322-327, 1983.
[17] P. Turchin and W. T. Thoeny, "Quantifying dispersal of southern pine beetles with mark-recapture experiments and a diffusion model," Ecological Applications, vol. 3, no. 1, pp. 187-198, 1993.

[18] E. E. Holmes, "Are diffusion models too simple? A comparison with telegraph models of invasion," American Naturalist, vol. 142, no. 5, pp. 779-795, 1993.

[19] P. M. Kareiva and N. Shigesada, "Analyzing insect movement as a correlated random walk," Oecologia, vol. 56, no. $2-3$, pp. 234-238, 1983.

[20] V. S. Manoranjan and P. Van Den Driessche, "On a diffusion model for sterile insect release," Mathematical Biosciences, vol. 79, no. 2, pp. 199-208, 1986.

[21] H. J. Barclay, "Population models with the release of chemosterilants for pest Control," Journal of Applied Ecology, vol. 18, pp. 679-695, 1981.

[22] H. J. Barclay, "Mathematical models for the use of sterile insects," in Sterile Insect Technique. Principles and Practice in Area-Wide Integrated Pest Management, V. A. Dyck, J. Hendrichs, and A. S. Robinson, Eds., pp. 147-174, Springer, Dordrecht, The Netherlands, 2005.

[23] M. A. Lewis and P. Van den Driessche, "Waves of extinction from sterile insect release," Mathematical Biosciences, vol. 116, no. 2, pp. 221-247, 1993.

[24] H. Caswell, Matrix Population Models: Construction, Analysis and Interpretation, Sinauer, Sunderland, Mass, USA, 2001.

[25] P. Yu, T. Habtemariam, D. Oryang, M. Obasa, D. Nganwa, and V. Robnett, "Stochastic model of spatial spread and control of tsetse flies (Diptera: Muscidae)," Environmental Entomology, vol. 25, no. 1, pp. 78-84, 1996.

[26] J. W. Dean, B. R. Williamson, and R. J. Phelps, "Bahavioural studies of Glossina morsitans Westw. Using tantalum-182," Bulletin of Entomological Research, vol. 58, pp. 764-771, 1968.

[27] C. H. N. Jackson, "The analysis of tsetse fly population III," Annals of Eugenics, vol. 14, pp. 91-108, 1948.

[28] E. F. Knipling, "Possibilities of insect control or eradication through the use of sexually sterile males," Journal of Economic Entomology, vol. 48, pp. 459-462, 1955.

[29] A. S. Robinson and G. Hooper, Eds., World Crop Pests, 3A. Fruit Flies, Their Biology, Natural Enemies and Control, Elsevier, New York, NY, USA, 1989.

[30] A. S. Robinson and G. Hooper, Eds., World Crop Pests, 3B. Fruit Flies, Their Biology, Natural Enemies and Control, Elsevier, New York, NY, USA, 1989.

[31] A. M. Wakid and A. Shoukry, "Dispersal and flight range of the Mediterranean fruit fly, Ceratitis capitata Wied. In Egypt," Zeitschrift fur Angewante Entomologie, vol. 81, pp. 214-218, 1976.

[32] T. T. Y. Wong, L. C. Whitehand, R. M. Kobayashi, K. Ohinata, N. Tanaka, and E. J. Harris, "Mediterranean fruit fly: dispersal of wild and irradiated and untreated laboratory-reared males," Environmental Entomology, vol. 11, pp. 339-343, 1982.

[33] R. E. Plant and R. T. Cunningham, "Analyses of the dispersal of sterile Mediterranean fruit flies (Diptera: Tephretidae) released from a point source," Environmental Entomology, vol. 20, pp. 1493-1503, 1991.

[34] A. Meats, C. J. Smallridge, and B. C. Dominiak, "Dispersion theory and the sterile insect technique: application to two species of fruit fly," Entomologia Experimentalis et Applicata, vol. 119, no. 3, pp. 247-254, 2006.

[35] A. Meats and C. J. Smallridge, "Short- and long-range dispersal of medfly, Ceratitis capitata (Dipt., Tephritidae), and its invasive potential," Journal of Applied Entomology, vol. 131, no. 8, pp. 518-523, 2007. 
[36] M. Aluja and P. Liedo, Eds., Fruit Flies. Biology and Management, Springer, New York, NY, USA, 1993.

[37] B. A. McPheron and G. J. Steck, Fruit Fly Pests: A World Assessment of Their Biology and Management, St. Lucie Press, Delray Beach, Fla, USA, 1996.

[38] R. Brightwell, R. D. Dransfield, and B. G. Williams, "Factors affecting seasonal dispersal of the tsetse flies Glossina pallidipes and G. longipennis (Diptera: Glossinidae) at Nguruman, south-west Kenya," Bulletin of Entomological Research, vol. 82, no. 2, pp. 167-182, 1992.

[39] A. Challier, "The ecology of tsetse (Glossina spp) (Diptera: Glossinidae): a review (1970-1981)," Insect Science and its Application, vol. 3, no. 2-3, pp. 97-143, 1982.

[40] P. A. Glasgow, The Distribution and Abundance of Tsetse, Permagon, Oxford, UK, 1963. 


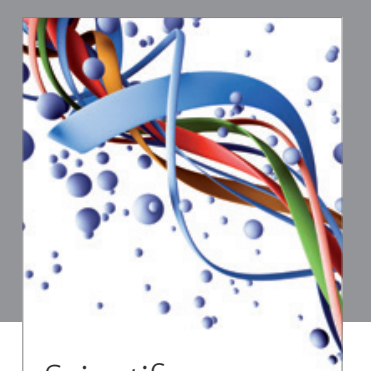

Scientifica
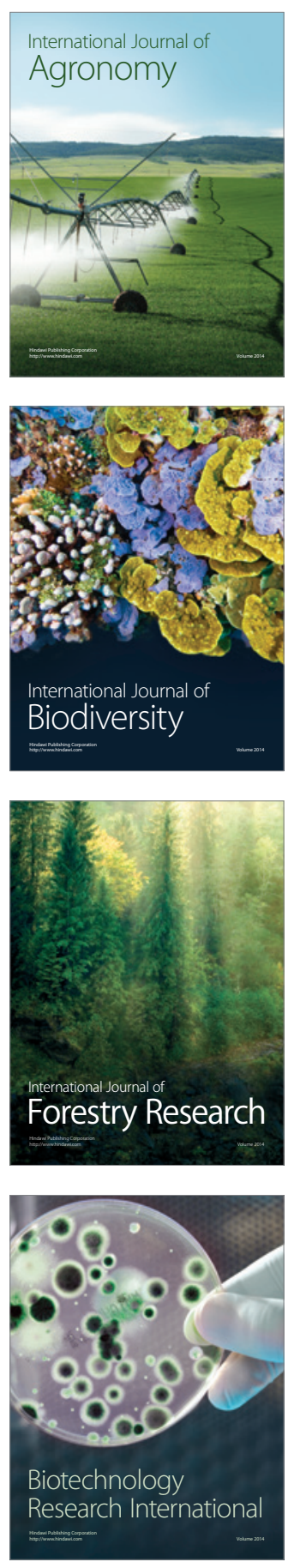
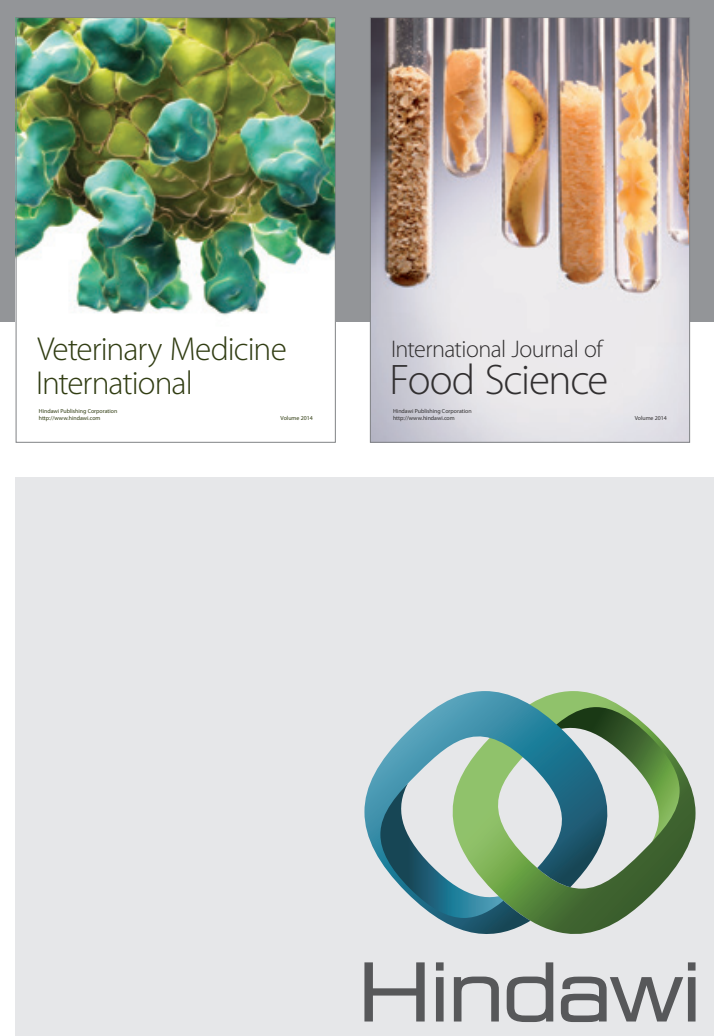

Submit your manuscripts at

http://www.hindawi.com
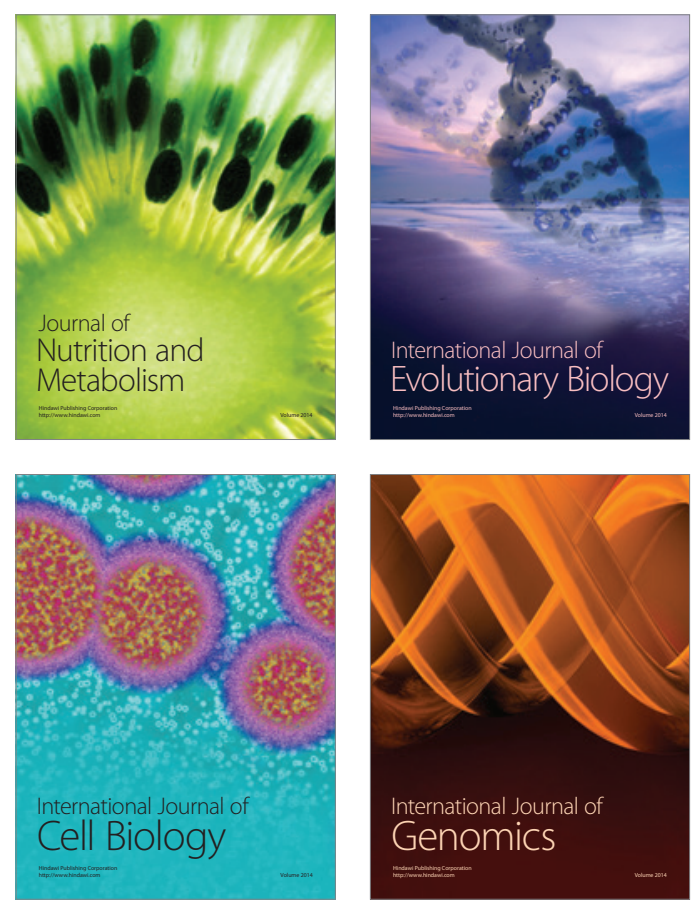
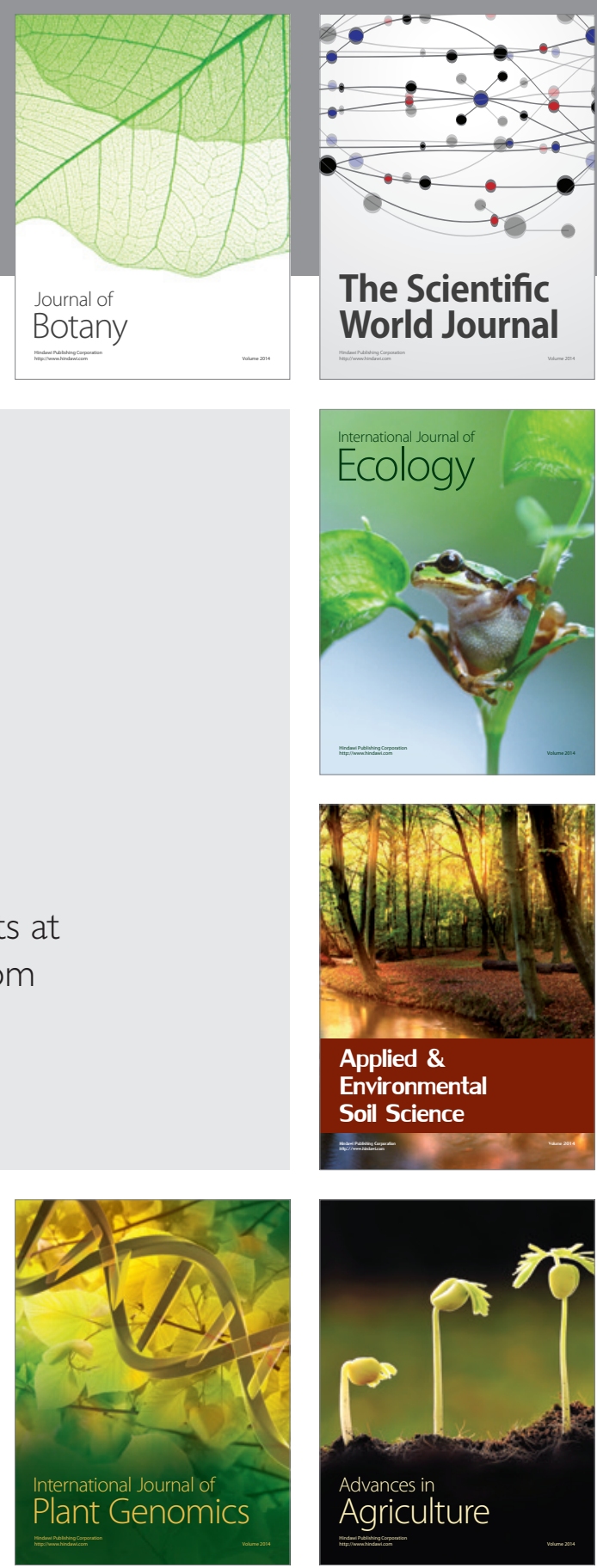

The Scientific World Journal
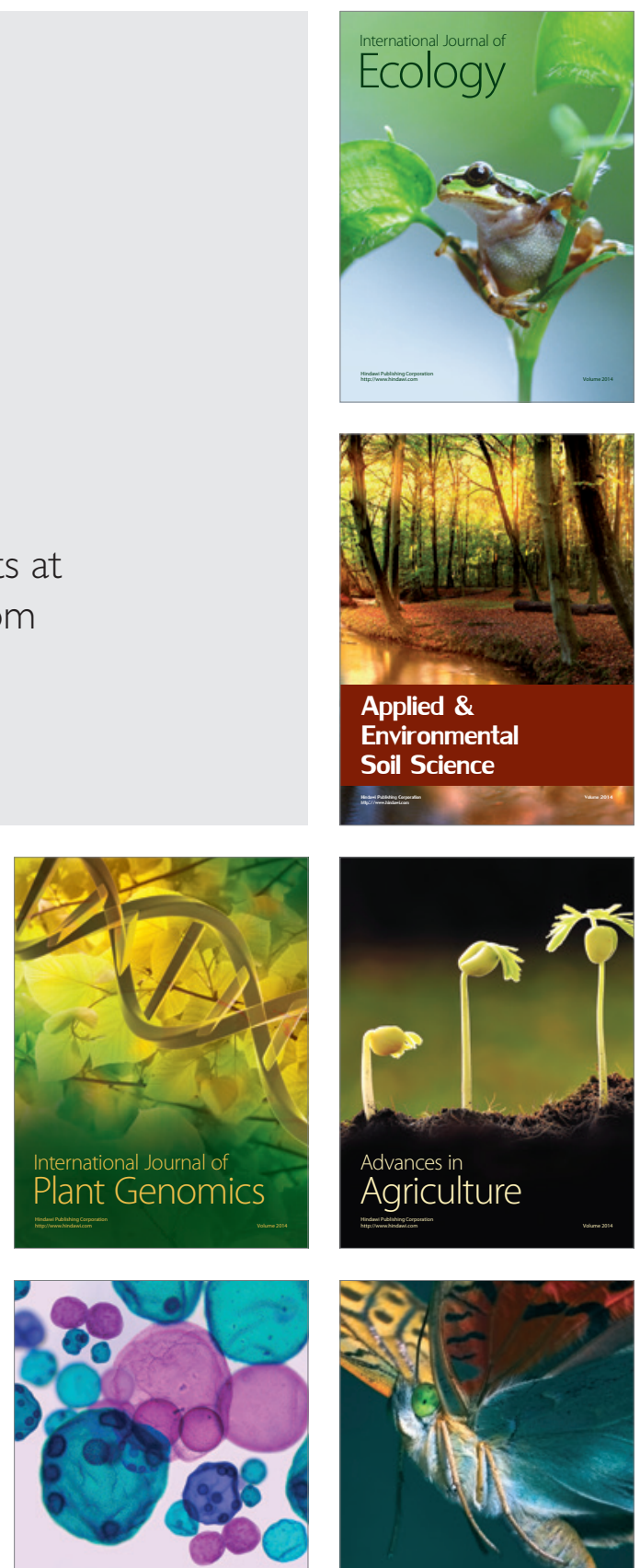

International Journal of Microbiology

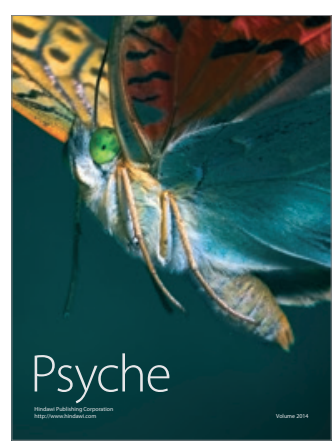

